Cite this: Phys. Chem. Chem. Phys., 2014, 16, 25694

Received 24th March 2014, Accepted 25th April 2014

DOI: $10.1039 / c 4 c p 01274 g$

www.rsc.org/pccp

\title{
Extension of the diffusion controlled electron transfer theory for intermittent fluorescence of quantum dots: inclusion of biexcitons and the difference of "on" and "off" time distributions
}

\author{
Zhaoyan Zhu and R. A. Marcus*
}

\begin{abstract}
The equations for the diffusion controlled electron transfer (DCET) theory of quantum dot blinking are extended to include biexcitons. In contrast to excitons, which undego resonant light to dark transitions, the biexcitons, having a much larger total energy, undergo a Fermi's Golden rule type transfer (many acceptance states). The latter immediately gives rise to an exponential tail for the light state, and it is explained why the dark state power law behavior is unaffected. Results are given for both continuous and pulsed excitation. The typical $-3 / 2$ power law for the light state at low light intensities, and for the dark state at all intensities, as well as dependence of the exponential tail on the square of the light intensity, and a decrease of the power in the power law for the light state from $-3 / 2$ to less negative values with increasing light intensity are all consistent with the theory. The desirability of measuring the dependence of the spectral diffusion coefficient on light intensity at room temperature as a test of several aspects of the theory is noted.
\end{abstract}

\section{Introduction}

Single molecule spectroscopy is a powerful and sensitive technique that permits the investigation of spatially heterogeneous samples one at a time and reveals phenomena masked by ensemble averaging. It is also complementary to ensemble studies. $^{1,2}$ It has been widely used to study the fluorescence of single quantum dots, ${ }^{1,3-16}$ single fluorophores in porous silicon, ${ }^{17}$ single polymer segments, ${ }^{18}$ light harvesting complexes, ${ }^{19}$ fluorescent proteins, ${ }^{20,21}$ and single dye molecules on various surfaces. ${ }^{22-29}$

The interesting phenomenon of blinking or fluorescence intermittency has been observed in which abrupt transitions occur between alternating episodes of absorption of light and fluorescence recycling is followed by sustained periods of darkness where no light is emitted. Numerous experiments have been performed since the first observation ${ }^{3}$ of the fluorescence blinking of quantum dots. ${ }^{1,7-16}$ Memory in subsequent fluorescence or dark episodes ${ }^{30}$ and electric field modulation of fluorescence $^{31}$ have been observed. Several models have been proposed to explain the phenomenon. ${ }^{2,32-38}$

In the present paper we extend a reaction diffusion differential equation to include biexcitons, prompted by recent experimental results. $^{39-46}$ The theory now contains two different mechanisms

Noyes Laboratory of Chemical Physics, California Institute of Technology, Pasadena, California 91125, USA. E-mail: ram@caltech.edu; Fax: +1 626792 8485; Tel: +16263956566 for the intermittency, resonant and Fermi's Golden rule, depending in the energy, and also now explaining why the light ("on”) state shows, typically, on the average at low light intensity an $\sim-3 / 2$ power law ${ }^{1,7-16}$ with an exponential tail that depends on the light intensity while the dark ("off") state only shows the $\sim-3 / 2$ power law, even under these high light intensity conditions.

Until now we have had a differential equation that treats the common $-3 / 2$ power law and an argument in the literature as to how biexcitons give rise to an exponential tail. This interesting "patchwork" is now replaced by a unified treatment, a differential equation for the light state and one for the dark state and their solution. A physical reason, based on amount of excess energy, is given as to why there are two forms of change of state, one that is a resonant transition $\left(\sim 0.3 \mathrm{eV}^{38,47}\right)$ and the other is a Fermi's Golden rule transition $\left(\sim 2.2 \mathrm{eV}^{41}\right)$. What emerges from the solution of the differential equations, understood in physical terms, is an explanation of why the light state but not the dark state shows an exponential tail. Before now one had to assume some not understood difference in diffusion constants for the explanation, an explanation that also would not have explained the intensity dependence of the exponential tail.

The paper is organized as follows: in Section 2, the previous Diffusion Controlled Electron Transfer (DCET) model is briefly summarized. The present extended DCET model with biexcitons is introduced there and the equation for the bright population density change with time is derived for both the continuous 
(Section 2.3) and pulsed laser excitation (Section 2.4) cases and solved for the survival probability. The equations of dark states population are also derived and the solution is given there. In Section 3, the quadratic dependence of the exponential tail on the excitation power, the difference between the bright and dark cycling, the dependence of the linear portion of the log-log plot for the "on" state on the light intensity and other phenomena are discussed. Concluding remarks are given in Section 4.

\section{Theory}

\subsection{DCET model}

The existence of an approximately $-3 / 2$ power law ${ }^{1,6-16}$ for the blinking has suggested that diffusion (a spectral diffusion) is involved in the blinking. ${ }^{34,48}$ Subsequently, a diffusion controlled electron transfer (DCET) equation was proposed ${ }^{2,38,47}$ to treat the intermittency. The diffusion considered here differs from that discussed in ref. 49, which considers diffusion of electrons rather than "spectral diffusion". A DCET mechanism is assumed to govern the charge transfer reactions between an "on" state $|e\rangle$ of the quantum dot and a charge-separated state $|d\rangle$ there (Fig. 1). The latter appears dark due to a fast Auger relaxation of an excited state $\left|d^{*}\right\rangle \cdot{ }^{3,6,8-10,13}$ That relaxation dominates any fluorescence. ${ }^{50}$ State $|d\rangle$ is a long-lived dark trapped state. However, what the theory did not do, and what we do in this paper, is to explain why the "off" state only shows the power law, whereas the "on" state has an exponential tail in addition to its power law and give and solve differential equations for these two states.

\subsection{DCET model with biexcitons}

We adopt the diffusion controlled electron transfer mechanism where the equations are given in ref. 38 , and to it we add a quite different mechanism for including biexcitons, different because of their high energy, and so add a term in the DCET equation containing this reaction step. In the present article we add biexcitons, while multi excitons will be included in a later paper. With biexciton generation, the previous fourlevel system ${ }^{38}$ is generalized to now include the new bright species, biexcitons.

We denote the ground state by $|g\rangle$, the excited state (one exciton) by $|\mathrm{e}\rangle$, the biexciton by $|\mathrm{b}\rangle$, the trapped state (the dark state) by $|d\rangle$ and the excited dark state by $\left|d^{*}\right\rangle$. These symbols each denote the state of the entire QD and not just part of it.
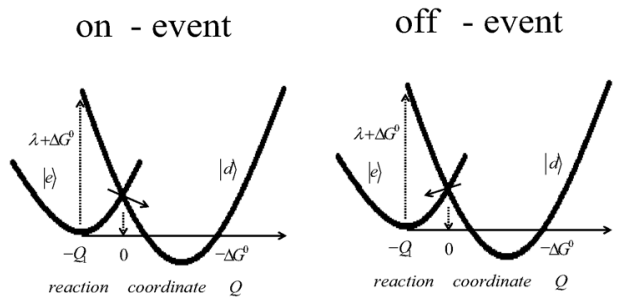

Fig. 1 Diffusion on the parabolic potential surfaces $|l\rangle$ and $|d\rangle$ across a sink at the energy-level crossing governs the intermittency phenomenon (corresponding to Fig. 2(b) from ref. 38).
The assumed reaction mechanism can be written as follows:

Reaction step Rate constant

For a bright QD:

$|\mathrm{g}\rangle+h \nu \rightarrow|\mathrm{e}\rangle$

$|\mathrm{e}\rangle \rightarrow|\mathrm{g}\rangle$

$I_{\text {ge }}$

$k_{\text {eg }}$

$|\mathrm{e}\rangle+h \nu \rightarrow|\mathrm{b}\rangle$

$I_{\mathrm{eb}}$

$k_{\text {ed }}$

$|e\rangle \rightarrow|d\rangle$

$k_{\text {be }}$

$|\mathrm{b}\rangle \rightarrow|\mathrm{e}\rangle$

$k_{\mathrm{bd}^{\prime}}$

$|\mathrm{b}\rangle \rightarrow\left|\mathrm{d}^{\prime}\right\rangle$

For a dark QD: we have

$\begin{array}{ll}|\mathrm{d}\rangle+h \nu \rightarrow\left|\mathrm{d}^{*}\right\rangle & I_{\mathrm{dd}^{*}} \\ \left|\mathrm{~d}^{*}\right\rangle \rightarrow|\mathrm{e}\rangle & k_{\mathrm{d}^{*} \mathrm{e}} \\ \left|\mathrm{d}^{*}\right\rangle \rightarrow|\mathrm{d}\rangle & k_{\mathrm{d}^{*} \mathrm{~d}} \\ \left|\mathrm{~d}^{\prime}\right\rangle \rightarrow|\mathrm{d}\rangle & k_{\mathrm{d}^{\prime} \mathrm{d}}\end{array}$

The state $|d\rangle$ is the lowest energy dark ground state, $\left|\mathrm{d}^{*}\right\rangle$ is a dark state with an extra exciton and $\left|\mathrm{d}^{\prime}\right\rangle$ is a higher energy dark state. The $k_{\text {eg }}$ and $k_{\mathrm{be}}$ are both a sum of the radiative and nonradiative rate constants, while $k_{\mathrm{bd}^{\prime}}$ is an Auger assisted ionization rate constant to form the dark state, ${ }^{51} k_{\mathrm{d}^{*} \mathrm{e}}$ is an Auger-assisted rate constant to form the bright state, ${ }^{47}$ and $k_{\mathrm{d}^{\prime} \mathrm{d}}$ is a fast relaxation process. The pulse duration of the commonly used pulse lasers is on the order of 1 ps to 100 ps. $^{41,52}$ The Auger process occurs in similar time range. ${ }^{42}$ Even after an Auger assisted ionization, a dark QD is still in the dark cycling, unlike the Auger kinetics in a bright QD.

\subsection{Continuous laser excitation}

Under continuous (cw) laser excitation the bright cycling equations contain the added kinetic step and are given by

$$
\begin{gathered}
\frac{\partial \rho_{\mathrm{g}}(Q, t)}{\partial t}=k_{\mathrm{eg}} \rho_{\mathrm{e}}(Q, t)-I_{\mathrm{ge}} \rho_{\mathrm{g}}(Q, t) \\
\frac{\partial \rho_{\mathrm{e}}(Q, t)}{\partial t}=I_{\mathrm{ge}} \rho_{\mathrm{g}}(Q, t)+L_{\mathrm{e}} \rho_{\mathrm{e}}(Q, t)+k_{\mathrm{be}} \rho_{\mathrm{b}}(Q, t) \\
-\left(k_{\mathrm{eg}}+I_{\mathrm{eb}}\right) \rho_{\mathrm{e}}(Q, t)-k_{\mathrm{ed}} \delta(Q) \rho_{\mathrm{e}}(Q, t), \\
\frac{\partial \rho_{\mathrm{b}}(Q, t)}{\partial t}=I_{\mathrm{eb}} \rho_{\mathrm{e}}(Q, t)+L_{\mathrm{b}} \rho_{\mathrm{b}}(Q, t)-\left(k_{\mathrm{be}}+k_{\mathrm{bd}^{\prime}}\right) \rho_{\mathrm{b}}(Q, t),
\end{gathered}
$$

where $L_{\mathrm{e}}$ is the diffusion operator in state $|\mathrm{e}\rangle$ and $L_{\mathrm{b}}$ is the diffusion operator for the biexciton state. From ref. 1 we know that a dark state is dark for an hour when there is no light. Our interpretation of this result is two-fold: the dark to bright transition is in the time period of the experiments a lightinduced Auger transition, and also the dark state doesn't diffuse in the absence of light (otherwise it would reach the intersection of the potential energy curves) and the ground state doesn't diffuse. The remaining symbols are the reaction rate constants shown in the reaction scheme given above. We have

$$
L_{\mathrm{e}} \equiv D_{\mathrm{e}} \frac{\partial}{\partial Q}\left[\frac{\partial}{\partial Q}+\frac{1}{k_{\mathrm{B}} T} \frac{\partial}{\partial Q} U_{\mathrm{e}}(Q)\right]
$$

where $D_{\mathrm{e}}$ is the diffusion constant for a structural, e.g., spectral, diffusion and $U_{\mathrm{e}}(Q)$ is the potential energy (more precisely 
a free energy curve $)^{38}$ as a function of a generalized coordinate $Q .{ }^{53,54}$

Unlike the resonant transition terms in eqn (2) given by the delta function (Fig. 1), we assume that because of the large energy present in the biexciton state an electron or perhaps even the more localized hole can be ejected to any site outside the core $\mathrm{QD}$, a process treated in the present derivation by Fermi's Golden rule and later leading to the exponential factor in the decay of the bright states, as seen in eqn (22) below. Due to fast Auger processes the lifetime of biexcitons is much shorter than the one of single exciton so we neglect any structural diffusion, the $L_{\mathrm{b}} \rho_{\mathrm{b}}$ term in eqn (3) in the biexciton states during the time available.

In the meanwhile, the dark cycling equations remain the same as in ref. 38 ,

$$
\begin{gathered}
\frac{\partial \rho_{\mathrm{d}}(Q, t)}{\partial t}=k_{\mathrm{d}^{*} \mathrm{~d}} \rho_{\mathrm{d}^{*}}(Q, t)-I_{\mathrm{d}^{*}} \rho_{\mathrm{d}}(Q, t), \\
\frac{\partial \rho_{\mathrm{d}^{*}}(Q, t)}{\partial t}=L_{\mathrm{d}^{*}} \rho_{\mathrm{d}^{*}}(Q, t)+I_{\mathrm{dd}^{*}} \rho_{\mathrm{d}}(Q, t) \\
-k_{\mathrm{d}^{*} \mathrm{~d}} \rho_{\mathrm{d}^{*}}(Q, t)-k_{\mathrm{d}^{*} \mathrm{e}} \delta(Q) \rho_{\mathrm{d}^{*}}(Q, t),
\end{gathered}
$$

where $L_{\mathrm{d}^{*}}$ is the diffusion operator in state $\left|\mathrm{d}^{*}\right\rangle$ and other symbols are reaction rate constants shown in the table. Since $\left|d^{\prime}\right\rangle$ all goes back to $|d\rangle$ the instantaneous contribution of $\rho_{\mathrm{d}^{\prime}}$ is negligible. Biexciton and multi exciton terms are not specifically included here because pumping to and relaxation from biexciton and multi exciton states is still in the dark cycling and plays no role in the dark to bright electron transfer reaction. In the present model the diffusion is stimulated by each absorption of light so a stepwise diffusion occurs just with light and so occurs in the $|e\rangle,|b\rangle$ and $\left|d^{*}\right\rangle$ states but not in the $|g\rangle$ and $|d\rangle$ states. So there is no $L_{\mathrm{d}}$ in eqn (5) since in the theory there is no spectral diffusion in this lowest dark state.

The sum of the three rate equations, eqn (1)-(3) yields the rate of change of the total "on" population $\rho_{\mathrm{g}}+\rho_{\mathrm{e}}+\rho_{\mathrm{b}}$, denoted by $\rho_{\mathrm{L}}$,

$$
\frac{\partial \rho_{\mathrm{L}}(Q, t)}{\partial t}=L_{\mathrm{e}} \rho_{\mathrm{e}}(Q, t)-k_{\mathrm{ed}} \delta(Q) \rho_{\mathrm{e}}(Q, t)-k_{\mathrm{bd}^{\prime}} \rho_{\mathrm{b}}(Q, t)
$$

In comparison the sum of the rate equations for the two dark states, eqn (5) and (6), yields the rate of change of the total "off", population $\rho_{\mathrm{d}}+\rho_{\mathrm{d}^{*}}$, denoted by $\rho_{\mathrm{D}}$ :

$$
\frac{\partial \rho_{\mathrm{D}}(Q, t)}{\partial t}=L_{\mathrm{d}^{*}} \rho_{\mathrm{d}^{*}}(Q, t)-k_{\mathrm{d}^{*} \mathrm{e}} \delta(Q) \rho_{\mathrm{d}^{*}}(Q, t),
$$

Here, the counterpart of the last term in eqn (7) which later is shown to result in the exponential tail in the solution is missing.

With $I_{\text {ge }}$ usually $<0.01 \mathrm{~ns}^{-1}$ (ref. $1,6-13,15,16$ and 27 ) $\ll$ $k_{\text {eg }}\left(\sim 0.1 \mathrm{~ns}^{-1}\right)^{52,55}$ and at any time $t \gg 1 / I_{\text {ge }}$, a quasiequilibrium is established between $|\mathrm{g}\rangle$ and $|\mathrm{e}\rangle$, yielding $k_{\mathrm{eg}} \rho_{\mathrm{e}}(Q, t) \approx I_{\mathrm{ge}} \rho_{\mathrm{g}}(Q, t)$. There is a similar quasiequilibrium between $|\mathrm{e}\rangle$ and $|\mathrm{b}\rangle$ and so $\rho_{\mathrm{b}} \approx \rho_{\mathrm{e}} I_{\mathrm{eb}} / k_{\mathrm{be}}$. If the absorption cross-section of a QD changes little with exciton generation, i.e., $I_{\mathrm{eb}}=I_{\mathrm{ge}}$, then

$$
\rho_{\mathrm{b}}=\frac{I_{\mathrm{ge}}}{k_{\mathrm{be}}} \rho_{\mathrm{e}},
$$

and

$$
\rho_{\mathrm{L}}=\frac{k_{\mathrm{eg}}}{I_{\mathrm{ge}}} \rho_{\mathrm{e}}+\rho_{\mathrm{e}}+\rho_{\mathrm{e}} \frac{I_{\mathrm{ge}}}{k_{\mathrm{be}}} \sim \frac{k_{\mathrm{eg}}}{I_{\mathrm{ge}}} \rho_{\mathrm{e}} .
$$

Eqn (7) can now be written as eqn (11) for cw

$$
\begin{aligned}
\frac{I_{\mathrm{ge}}}{k_{\mathrm{eg}}} \frac{\partial \rho_{\mathrm{L}}(Q, t)}{\partial t}= & L_{\mathrm{e}} \rho_{\mathrm{L}}(Q, t)-k_{\mathrm{ed}} \delta(Q) \rho_{\mathrm{L}}(Q, t) \\
& -\rho_{\mathrm{L}}(Q, t) I_{\mathrm{ge}} \frac{k_{\mathrm{bd}^{\prime}}}{k_{\mathrm{be}}} .
\end{aligned}
$$

With a similar quasiequilibrium between $|\mathrm{d}\rangle$ and $\left|\mathrm{d}^{*}\right\rangle, \rho_{\mathrm{d}} \approx$ $\rho_{\mathrm{d}^{*}} k_{\mathrm{d}^{*} \mathrm{~d}} / I_{\mathrm{dd}^{*}}$ and $\rho_{\mathrm{D}}=\rho_{\mathrm{d}^{*}} k_{\mathrm{d}^{*} \mathrm{~d}} / I_{\mathrm{dd}^{*}}+\rho_{\mathrm{d}^{*}} \approx \rho_{\mathrm{d}^{*}} k_{\mathrm{d}^{*} \mathrm{~d}} / I_{\mathrm{dd}^{*}}$, and eqn (8) becomes

$$
\frac{I_{\mathrm{dd}^{*}}}{k_{\mathrm{d}^{*} \mathrm{~d}}} \frac{\partial \rho_{\mathrm{D}}(Q, t)}{\partial t}=L_{\mathrm{d}^{*}} \rho_{\mathrm{D}}(Q, t)-k_{\mathrm{d}^{*} \mathrm{e}} \delta(Q) \rho_{\mathrm{D}}(Q, t) .
$$

The solution of eqn (11) for $\rho_{\mathrm{L}}$ and (12) for $\rho_{\mathrm{D}}$ is given in Section 2.5.

\subsection{Pulsed laser excitation}

For pulsed laser excitation, there are two time frames, one arising at the start of the experiment, $t$, and the other, the time between consecutive pulses counting from the start of every pulse, $t_{\mathrm{r}}$, where $0 \leq t_{\mathrm{r}}<T, T$ being the interval between consecutive pulses. Fluorescence lifetime, biexciton and multi exciton lifetime and other transient properties occur within the $t_{\mathrm{r}}$ frame while the "on" and "off" time distribution occurs in the $t$ frame. With $T$ (typically $200 \mathrm{ns)} \mathrm{much} \mathrm{greater} \mathrm{than} \mathrm{an}$ exciton lifetime ( $\sim 10 \mathrm{~ns})$, before the next pulse starts, all bright exciton states have relaxed either back to ground state or transferred to dark states. The density of biexcitons transferred to dark states in every pulse, $p_{\text {lost }}(\mathrm{b})$, is

$$
p_{\text {lost }}(\mathrm{b})=P_{\text {ionb }} \rho_{\mathrm{b}}(Q, t),
$$

where $P_{\text {ionb }}$ is the ionization efficiency of a biexciton. If we adopt $T$, the interval between successive incident light pulses, as the unit of $t$, then $p_{\text {lost }}(\mathrm{b})$ is the rate of biexcitons transferred to dark states and we then have

$$
\begin{aligned}
\frac{\partial \rho_{\mathrm{L}}(Q, t)}{\partial t}= & L_{\mathrm{e}} \rho_{\mathrm{e}}(Q, t) \frac{\tau_{\mathrm{x}}}{T}-k_{\mathrm{ed}} \delta(Q) \rho_{\mathrm{e}}(Q, t) \frac{\tau_{\mathrm{x}}}{T} \\
& -P_{\text {ionb }} \rho_{\mathrm{b}}(Q, t),
\end{aligned}
$$

where $\tau_{\mathrm{x}}$ is the single exciton lifetime, while for the dark states we have

$$
\frac{\partial \rho_{\mathrm{D}}(Q, t)}{\partial t}=L_{\mathrm{d}^{*}} \rho_{\mathrm{d}^{*}}(Q, t) \frac{\tau_{\mathrm{x}}}{T}-k_{\mathrm{d}^{*} \mathrm{e}} \delta(Q) \rho_{\mathrm{d}^{*}}(Q, t) \frac{\tau_{\mathrm{x}}}{T},
$$

Treating the absorption of photons as consisting of independent events, we then have a Poisson distribution. In every single pulse, the probability of absorbing of $m$ incoming photons by a QD forming an $m$ exciton is

$$
P(m)=\mathrm{e}^{-\left\langle M_{\mathrm{x}}\right\rangle} \frac{\left\langle M_{\mathrm{x}}\right\rangle^{m}}{m !}
$$

where $\left\langle M_{\mathrm{x}}\right\rangle$ is the average exciton number formed in a QD per pulse. Here, we only consider ground states, single excitons 
and biexcitons. Then $\rho_{\mathrm{L}}(Q, t)=\rho_{\mathrm{g}}(Q, t)+\rho_{\mathrm{e}}(Q, t)+\rho_{\mathrm{b}}(Q, t)$ and eqn (14) can be rewritten as

$$
\begin{aligned}
& \frac{\left\langle M_{\mathrm{x}}\right\rangle}{1+\left\langle M_{\mathrm{x}}\right\rangle+\left\langle M_{\mathrm{x}}\right\rangle^{2} / 2} \frac{\partial \rho_{\mathrm{L}}(Q, t)}{\partial t}= \\
& L_{\mathrm{e}} \rho_{\mathrm{L}}(Q, t) \frac{\tau_{\mathrm{x}}}{T}-k_{\mathrm{ed}} \delta(Q) \rho_{\mathrm{L}}(Q, t) \frac{\tau_{\mathrm{x}}}{T}-\rho_{\mathrm{L}}(Q, t) P_{\text {ionb }} \frac{\left\langle M_{\mathrm{x}}\right\rangle}{2} .
\end{aligned}
$$

For the dark states $\rho_{\mathrm{D}}(Q, t)=\rho_{\mathrm{d}}(Q, t)+\rho_{\mathrm{d}^{*}}(Q, t)$ and eqn (15) becomes

$$
\frac{\left\langle M_{\mathrm{x}}\right\rangle}{1+\left\langle M_{\mathrm{x}}\right\rangle} \frac{\partial \rho_{\mathrm{D}}(Q, t)}{\partial t}=L_{\mathrm{d}^{*}} \rho_{\mathrm{D}}(Q, t) \frac{\tau_{\mathrm{x}}}{T}-k_{\mathrm{d}^{*} \mathrm{e}} \delta(Q) \rho_{\mathrm{D}}(Q, t) \frac{\tau_{\mathrm{x}}}{T} .
$$

\subsection{Solution}

Without the last term eqn (11) and (17) (and in the case of eqn (17) without the $\left\langle M_{\mathrm{x}}\right\rangle^{2} / 2$ term) are similar to the eq (4a) obtained by Tang and Marcus, ${ }^{38}$ as are eqn (12) and (18) for the dark states. Comparing eqn (11) and (17) with eq (4a) we then see that the solution of eqn (11) and (17) equals that of eq (4a) multiplied by $\mathrm{e}^{-k_{\mathrm{c}} t}$, where $k_{\mathrm{c}}=I_{\mathrm{ge}^{2}}{ }^{2} k_{\mathrm{bd}^{\prime}} /\left[k_{\mathrm{eg}} k_{\mathrm{be}}\right]$ and $k_{\mathrm{c}}=$ $P_{\text {ionb }}\left\langle M_{\mathrm{x}}\right\rangle^{2} /\left[2\left(1+\left\langle M_{\mathrm{x}}\right\rangle+\left\langle M_{\mathrm{x}}\right\rangle^{2} / 2\right)\right]$ for eqn (11) and (17), respectively while for the dark states the solution of eqn (11) and (17) is the same as the solution of eq (4a). Here, we consider the case that ${ }^{41}$ during a pulse excitation there is no accumulation of excitons from previous pulses, i.e., all bright exciton states have relaxed either back to the ground state or transformed to dark states. The integration of the probability density function $\rho_{\mathrm{L}}(Q, t)$ over $Q$ gives the survival probability at time $t$ for an "on" state that started at $t=0$. Treating the survival probability for "on" as a step function, noting that the derivative of a step function is a $\delta$ function, the derivative gives the time at which a change occurs from "on" to "off", so giving the waiting-time distribution $P_{\mathrm{L}}(t)$. This $P_{\mathrm{L}}(t)$ for a quantum dot, defined in eqn (19) as the derivative of the survival probability, is the probability of a QD that has been in the "on" cycling for $t$ and transfers to an "off" cycling during $\mathrm{d} t$ per unit $\mathrm{d} t$. It is given by

$$
P_{\mathrm{L}}(t)=-\frac{\mathrm{d}}{\mathrm{d} t} \int_{-\infty}^{\infty} \mathrm{d} Q \rho_{\mathrm{L}}(Q, t),
$$

where $Q$ is the reaction coordinate. When the quantum dot is in the vicinity of the intersection of the relevant electronic state potential energy surfaces, ${ }^{56}$ the effect of the slopes of the corresponding potential energy surfaces $U_{11}(Q)^{38,57}$ on the dynamics will be assumed to be small $(t<1 / \Gamma$, in the notation of ref. 2 and 38). The diffusion operator $L_{11}$ defined in eqn (4) can then be approximated as $D_{11} \partial^{2} / \partial Q^{2}$ which is the no-forced diffusion term. We use the Green function $\operatorname{method}^{58}$ to solve eqn (17) with the free energy derivatives ${ }^{2,38}$ with respect to $Q$ absent and, as before, ${ }^{38}$ obtain a closed form solution in Laplace transform space. The solution for $\rho_{\mathrm{L}}(Q, t)$ and hence for $P_{\mathrm{L}}(t)$ is given by

$$
P_{\mathrm{L}}(t)=\frac{1+2 k_{\mathrm{c}} t}{\sqrt{\pi t_{\mathrm{c}} t}}\left[1-\sqrt{\frac{\pi t}{t_{\mathrm{c}}}} \mathrm{e}^{t / t_{\mathrm{c}}} \operatorname{erfc}\left(\sqrt{\frac{t}{t_{\mathrm{c}}}}\right)\right] \mathrm{e}^{-k_{\mathrm{c}} t}
$$

with the limiting forms (21) and (22) for $t \ll t_{\mathrm{c}}$ and $t \gg t_{\mathrm{c}}$.

$$
P_{\mathrm{L}}(t) \approx \frac{1}{\sqrt{\pi t_{\mathrm{c}}} t}, \quad t \ll t_{\mathrm{c}}
$$

and

$$
P_{\mathrm{L}}(t) \approx \frac{\sqrt{t_{\mathrm{c}}}}{\sqrt{4 \pi t^{3}}} \mathrm{e}^{-k_{\mathrm{c}} t}, \quad t \gg t_{\mathrm{c}}
$$

where $t_{\mathrm{c}}$ is the critical time $\mathrm{e}^{2,38}$ in which the population has largely been depleted near the sink (time to set up a steadystate) due to disappearance into the sink at the crossing and

$$
t_{\mathrm{c}}=4 D_{\mathrm{e}} I_{\mathrm{ge}} /\left[k_{\mathrm{eg}} k_{\mathrm{ed}}^{2}\right]
$$

and

$$
t_{\mathrm{c}}=4 D_{\mathrm{e}} T\left\langle M_{\mathrm{x}}\right\rangle /\left[\tau_{\mathrm{x}}\left(1+\left\langle M_{\mathrm{x}}\right\rangle+\left\langle M_{\mathrm{x}}\right\rangle^{2} / 2\right) k_{\mathrm{ed}}^{2}\right]
$$

for eqn (11) and (17) respectively. For cw excitation,

$$
k_{\mathrm{c}}=I_{\mathrm{ge}}^{2} \frac{k_{\mathrm{bd}^{\prime}}}{k_{\mathrm{eg}} k_{\mathrm{be}}},
$$

and for pulsed excitation,

$$
k_{\mathrm{c}}=P_{\text {ionb }} \frac{\left\langle M_{\mathrm{x}}\right\rangle^{2}}{2\left(1+\left\langle M_{\mathrm{x}}\right\rangle+\left\langle M_{\mathrm{x}}\right\rangle^{2} / 2\right)} \sim P_{\text {ionb }}\left\langle M_{\mathrm{x}}\right\rangle^{2} / 2,
$$

where $\left\langle M_{\mathrm{x}}\right\rangle$ is the average exciton number formed in a QD per pulse. Comparing eqn (7) and (14), we can see that $k_{\mathrm{bd}}$ plays the role of $P_{\text {ionb }} I_{\mathrm{ge}}{ }^{2} /\left(k_{\mathrm{eg}} k_{\mathrm{be}}\right)$ and $\left\langle M_{\mathrm{x}}\right\rangle^{2} / 2$ are the ratio $\rho_{\mathrm{b}} / \rho_{\mathrm{L}}$, respectively, for the continuous and pulsed laser excitation.

In concluding this section, we make one further remark on eqn (22). The complete form of eqn (22) is

$$
P_{\mathrm{L}}(t) \approx \frac{\sqrt{t_{\mathrm{c}}}}{\sqrt{4 \pi t^{3}}} \mathrm{e}^{-k_{\mathrm{c}} t}\left(1+2 k_{\mathrm{c}} t\right), \quad t \gg t_{\mathrm{c}} .
$$

The second term in the parentheses in eqn (22) only contributes when at $t \sim 1 / k_{\mathrm{c}}$ and would contribute to the little rise from the power law (Fig. 3) at the beginning of the exponential tail. It is a very small effect and would be hard to see in experimental results. ${ }^{41,46}$ For the dark states the distribution is again given by eqn (20), with the $k_{\mathrm{c}}$ in eqn (21) and (22) equal to zero, and with the $t_{\mathrm{c}}$ given by eqn (28) and (29) for $\mathrm{cw}$ and pulsed laser excitation instead of eqn (23) and (24).

$$
\begin{gathered}
t_{\mathrm{c}}=4 D_{\mathrm{d}^{*} I_{\mathrm{dd}^{*}} /\left[k_{\mathrm{d}^{*} \mathrm{~d}} k_{\mathrm{d}^{*} \mathrm{e}}{ }^{2}\right]} \\
t_{\mathrm{c}}=4 D_{\mathrm{d}^{*}} T\left[1+\left\langle M_{\mathrm{x}}\right\rangle\right] /\left[\tau_{\mathrm{x}}\left\langle M_{\mathrm{x}}\right\rangle k_{\left.\mathrm{d}^{*} \mathrm{e}^{2}\right]}{ }^{2}\right]
\end{gathered}
$$

\section{Results and discussion}

\subsection{Absence of exponential decay of the dark cycling}

While the dark cycling is similar to the bright cycling, the difference is that in dark cycling the fluorescence is quenched by an Auger process. If there is an Auger-assisted ionization from the dark state with two extra excitons, then after ionization the QD is still in the dark cycling phase and not yet transformed to the bright cycling: there are now two extra charges of the same kind in the conduction band or in the valence band that 
can participate in the Auger process. The exponential decay seen in eqn (22) for the "on" state is absent in the "off" waitingtime distribution $P_{\mathrm{D}}(t)$, since the dark state remains a dark state after this transition.

\subsection{Quadratic dependence of the exponential tail of the "on" time distribution on the excitation power}

At an intermediate excitation intensity when the chance of multi exciton generation higher than a biexciton is small, only the ground, exciton and biexciton states need be considered, $\rho_{\mathrm{L}} / \rho_{\mathrm{e}} \sim k_{\mathrm{eg}} / I_{\mathrm{ge}}$ and $\left\langle M_{\mathrm{x}}\right\rangle$ is small but large enough to cause the exponential tail. The exponential for the "on" state in eqn (22) is $\exp \left(-k_{\mathrm{c}} t\right)$. For cw excitation, $k_{\mathrm{c}} \sim I_{\mathrm{ge}^{2}}{ }^{2} k_{\mathrm{bd}} /\left[k_{\mathrm{eg}} k_{\mathrm{be}}\right]$ and for pulsed excitation, $k_{\mathrm{c}} \sim P_{\text {ionb }}\left\langle M_{\mathrm{x}}\right\rangle^{2} / 2$. This result agrees with the quadratic dependence of the exponential tail on the excitation power, as in the present Fig. 2 and 3 and in Fig. 4 of ref. 41 . According to ref. 41 , for the 3 points in Fig. $2\left\langle M_{\mathrm{x}}\right\rangle$ is $\sim 0.1$ to 0.3 . When the excitation intensity increases and multi excitons have to be taken into account, and in general this quadratic dependence is not expected. The general case with multi exciton species taken into account will be treated in a later paper.

\subsection{Slope of power law at short time}

A prediction of the diffusion controlled electron transfer model for the power law is that the power at short times will be $-1 / 2$ instead of $-3 / 2$ at longer times and the transition happens at the critical time $t_{\mathrm{c}}$ as seen in eqn (21) and (22). A subsequent test of this prediction was made and the expected change of unity in the slope ${ }^{59}$ was observed. However, while the result is

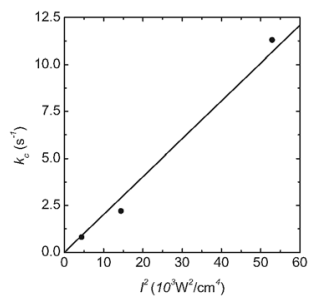

Fig. $2 k_{\mathrm{c}}$ vs. $I^{2}$ fitting where $l$ is laser intensity.

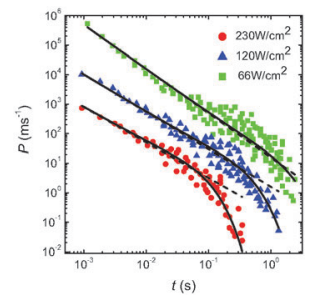

Fig. 3 On-time probability distributions measured under pulsed laser conditions at $\lambda_{\text {exc }}=434 \mathrm{~nm}$ and laser intensities of 230 (red $\boldsymbol{\bullet}$ ), 120 (blue $\boldsymbol{\Lambda}$ ), and $66 \mathrm{~W} \mathrm{~cm}^{-2}$ (green $\mathbf{a}$ ). The solid lines are fits to the data of power law with exponential cut-offs $k_{\mathrm{c}}\left(k_{\mathrm{c}}=11.3,2.2,0.8 \mathrm{~s}^{-1}\right.$, respectively) and bumps according to eqn (22) and the dashed lines are fits to the data only of power law. Here the slopes are not fixed at 3/2. The curves ${ }^{41}$ had been vertically shifted by unspecified amounts. Data reprinted with permission from ref. 41. Copyright (2009) American Chemical Society. supportive of the theory it doesn't confirm it since in principle it might have some other origin.

\subsection{Slope deviation from $-3 / 2$}

For the dark state the slope $m$ of the $\log P v s$. $\log t$ plot is $-3 / 2$ but for the light state at high intensities in Fig. $3 \mathrm{~m}$ is different (lower power) from $-3 / 2$ and this difference increases with increased light intensity. ${ }^{41}$ The mechanism of this light intensity dependence of the power law slope remains to be analyzed. One possibility is a binning effect on the $\log -\log$ plot. $^{60}$ If there is not a sharp distinction between "dark" and "light" for example, if there are different degrees of apparent brightness in the bright state due to fluctuations in numbers of bright periods within a bin, then the analysis would be more complex. In such a case, the trajectories would look "ragged" for a light state, instead of an ideal "picket fence" type trajectory. When possible, we have focused on systems that display the even height "picket fence" type trajectory rather than a "ragged" one, as in the trajectories shown in ref. 41 and 61 but not those in ref. 62 . In general, it would be helpful to decrease the bin size when analysing high excitation intensity experimental results and see if the power exponent converges to a constant value, signal intensity permitting.

Another possible origin is the change of the critical time $t_{\mathrm{c}}$ with a change of laser excitation intensity $I . t_{\mathrm{c}} \propto D_{\mathrm{e}} I_{\text {ge }}$. Since both $D_{\mathrm{e}}$ and $I_{\mathrm{ge}}$ increase with increasing $I, t_{\mathrm{c}}$ becomes larger and the probability of "on" events shorter than $t_{\mathrm{c}}$ (slope $-1 / 2$ as in eqn (21))becomes larger. If we assume $D_{\mathrm{e}} \propto I$ and $I_{\text {ge }} \propto I$, then $t_{\mathrm{c}} \propto I^{2}$. Scaling $t$ with $I^{2}$ (Fig. 4) one sees that the data points of the power law parts at the 3 different intensities in Fig. 3 merge and are fit with $t_{\mathrm{c}}=3 \mathrm{~ms}$ at $230 \mathrm{~W} \mathrm{~cm}^{-2}$ by eqn (20) without the exponential term. This $t_{\mathrm{c}}$ is comparable to results obtained at approximately the same absorption rate in ref. 59, a $t_{\mathrm{c}} \sim 5 \mathrm{~ms}$. Recently Bawendi and coworkers directly observed spectral diffusion dynamics in single CdSe-CdS QDs at low temperature. ${ }^{63}$ If diffusion constants can be obtained at room temperature this possibility can be tested.

The theory for the other experimental observations described earlier is independent of this possible explanation of this light intensity dependence of the initial linear slope in the experiments of ref. 41 .

\subsection{Nature of the trapped states}

Indirect evidence for a localized dark state with a localized charge trapped in a set of surface sites is seen in the experiment

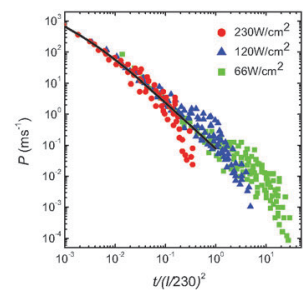

Fig. 4 On-time probability distributions with time $t$ scaled with excitation intensity $l^{2}$. Only the continuity of the linear portion of the plot should be examined. 
of Barbara and coworkers. ${ }^{31}$ Their result indicates that there is no uniform surface band, but rather that a localized charge is trapped at different surface sites at different times, there being perhaps a trap to trap diffusion of the charge on the surface. The Babara experiment doesn't determine the sign of the surface trapped state. Here, the "off" times are attributed to surface charge trapped states. Efros, Hermier and coworkers have measured the polarization-resolved PL spectra in magnetic fields in which the sign of the circular polarization degree indicates the sign of the resident charge to determine the extra charge ${ }^{64}$ in the core is an electron. Thus the dark state is formed when a hole transfers from an exciton state to a trap $|d\rangle$ off the valence band leaving an extra electron in the core. Guyot-Sionnest and coworkers showed that an $1 \mathrm{~S}_{\mathrm{e}}$ electron pumped to $1 \mathrm{P}_{\mathrm{e}}$ state has a large chance to tunneling to the surface. ${ }^{65}$ This is interpreted as the electron transfer discussed in ref. 47. In ref. 47 the state was assumed to be a state off the valence band. In the latter, the trapped state is a surface $\mathrm{Se}^{2-}$ prior to the transition that causes it to become a $\mathrm{Se}^{-}$after the transition, in the case of a CdSe QD. ${ }^{57}$ That is, it is a trapped hole off the valence band. Correspondingly, in this trapped state there is in the body of the QD an excess electron in the $1 \mathrm{P}_{\mathrm{e}}$ state. This view treats the trapped state as formed in an Auger process from a possible resonance to $1 \mathrm{P}_{\mathrm{e}}$ to $1 \mathrm{~S}_{\mathrm{e}}$ transition from a transition between a $1 \mathrm{~S}$ hole state in the valence band and a $\mathrm{Se}^{2-}$ surface state. For biexcitons, with two electrons in the $1 \mathrm{~S}_{\mathrm{e}}$ state and two holes in the $1 \mathrm{~S}_{\mathrm{h}}$ state, we have an Auger assisted ionization process in which one hole comes deeply from the valence band to the $\mathrm{Se}^{2-}$ ion and another hole annihilates one electron in the $1 \mathrm{~S}_{\mathrm{e}}$ state. In this trapped state with an excess electron in the core of the quantum dot when another electron is photo excited from the ground state to the conduction band Auger dominated nonradiative pathways can dominate the fluorescence. This oxidation by hole was evidenced recently in electrical charging experiments. ${ }^{66}$

\subsection{Other remarks}

A memory in consecutive bright or consecutive dark events has been reported by Stefani et al. ${ }^{30}$ It may now be due to successive trapping preferentially at or near a particularly favorable local site a dangling surface $\mathrm{Se}^{2-}$, for example, reflecting an expected heterogeneity in properties of the individual surface sites.

\section{Conclusions}

The equations for the DCET model have been extended so as to include biexcitons at the higher light intensities. The new partial differential equation modified from an earlier work by the inclusion of biexcitons provides an interpretation of the exponential cut-off of the power law time distribution of the "on" state of the single quantum dot fluorescence blinking process, the quadratic dependence of the exponential tail on the excitation intensity, and, particularly, the previously unexplained asymmetry between "on" and "off" states, only the former having an exponential tail in the observed time domain. Several other experiments including an effect of electric fields ${ }^{31}$ are also stated. ${ }^{30,59}$

\section{Acknowledgements}

It is a pleasure to acknowledge the support of this research by the Office of Naval Research, the Army Research Office and the James W. Glanville Foundation, and thank Prof. Peterson for providing his experimental data.

\section{References}

1 I. Chung and M. G. Bawendi, Phys. Rev. B: Condens. Matter Mater. Phys., 2004, 70, 165304.

2 J. Tang and R. A. Marcus, J. Chem. Phys., 2005, 123, 204511.

3 M. Nirmal, B. O. Dabbousi, M. G. Bawendi, J. J. Macklin, J. K. Trautman, T. D. Harris and L. E. Brus, Nature, 1996, 383, 802-804.

4 J. Tittel, W. Gohde, F. Koberling, A. Mews, A. Kornwski, H. Weller, A. Eychmuller and T. Basche, Ber. Bunsen-Ges., 1997, 101, 1626-1630.

5 U. Banin, M. Bruchez, A. P. Alivisatos, T. Ha, S. Weiss and D. S. Chemla, J. Chem. Phys., 1999, 110, 1195-1201.

6 M. Kuno, D. P. Fromm, H. F. Hamann, A. Gallagher and D. J. Nesbitt, J. Chem. Phys., 2000, 112, 3117-3120.

7 M. Kuno, D. P. Fromm, A. Gallagher, D. J. Nesbitt, O. I. Micic and A. Nozik, Nano Lett., 2001, 1, 557-564.

8 M. Kuno, D. P. Fromm, H. F. Hamann, A. Gallagher and D. J. Nesbitt, J. Chem. Phys., 2001, 115, 1028-1040.

9 K. T. Shimizu, R. G. Neuhauser, C. A. Leatherdale, S. A. Empedocles, W. K. Woo and M. G. Bawendi, Phys. Rev. B: Condens. Matter Mater. Phys., 2001, 63, 205316.

10 K. T. Shimizu, R. G. Neuhauser, C. A. Leatherdale, S. A. Empedocles, W. K. Woo and M. G. Bawendi, Phys. Rev. Lett., 2002, 89, 1117401.

11 X. Brkmann, J. P. Hermier, G. Messin, P. Desbiolles, J. P. Bouchaud and M. Dahan, Phys. Rev. Lett., 2003, 90, 120601.

12 R. Ververk, A. M. van Oijen and M. Orrit, Phys. Rev. B: Condens. Matter Mater. Phys., 2002, 66, 233202.

13 M. Kuno, D. P. Fromm, S. T. Johnson, A. Gallagher and D. J. Nesbitt, Phys. Rev. B: Condens. Matter Mater. Phys., 2003, 67, 125304.

14 S. Cui, T. Tachikawa, M. Fujitsuka and T. Majima, J. Phys. Chem. C, 2008, 112, 19625-19634.

15 S. Cui, T. Tachikawa, M. Fujitsuka and T. Majima, J. Phys. Chem. C, 2010, 114, 1217-1225.

16 S. Cui, T. Tachikawa, S. Tojo, M. Fujitsuka and T. Majima, J. Phys. Chem. C, 2011, 115, 1824-1830.

17 M. D. Mason, G. M. Credo, K. D. Weston and S. K. Burrato, Phys. Rev. Lett., 1998, 80, 5405-5408.

18 D. A. Vandenbout, W. T. Yip, D. H. Hu, D. K. Fu, T. M. Swager and P. F. Barbara, Science, 1997, 277, 1074-1077.

19 M. A. Bopp, Y. Jia, L. Li, R. Cogdell and R. M. Hochstrasser, Proc. Natl. Acad. Sci. U. S. A., 1997, 94, 10630-10635.

20 R. M. Dickson, A. B. Cubitt, R. Y. Tsien and W. E. Moerner, Nature, 1997, 388, 355-358.

21 E. J. G. Peterman, S. Brasselet and W. E. Moerner, J. Phys. Chem. A, 1999, 103, 10553-10560.

22 H. P. Lu and X. S. Xie, Nature, 1997, 385, 143-146. 
23 T. Ha, T. Enderle, D. S. Chemla, P. R. Selvin and S. Weiss, Chem. Phys. Lett., 1997, 271, 1-5.

24 K. D. Weston, P. J. Carson, H. Metiu and S. K. Buratto, J. Chem. Phys., 1998, 109, 7474-7485.

25 E. K. L. Yeow, S. M. Melnikov, T. D. M. Bell, F. C. De Schryver and J. Hofkens, J. Phys. Chem. A, 2006, 110, 1726-1734.

26 T. Tachikawa, S. Cui, S. Tojo, M. Fujitsuka and T. Majima, Chem. Phys. Lett., 2007, 443, 313-318.

27 T. Tachikawa, T. Ishigki, J. Li, M. Fujitsuka and T. Majima, Angew. Chem., Int. Ed., 2008, 47, 5348-5352.

28 L. K. Schirra, B. S. Tachett, M. L. Blumenfeld and O. L. A. Monti, J. Chem. Phys., 2009, 131, 124702.

29 Z. Bian, T. Tachikawa, S. Cui, S. Tojo, M. Fujitsuka and T. Majima, Chem. Sci., 2012, 3, 370-379.

30 F. Stefani, X. Zhong, W. Knoll, M. Han and M. Kreiter, New J. Phys., 2005, 7, 197.

31 S. Park, S. Link, W. L. Miller, A. Gesquiere and P. Barbara, Chem. Phys., 2007, 341, 169-174.

32 J. Wang and P. Wolynes, J. Chem. Phys., 1999, 110, 4812-4819.

33 M. Boguna, A. M. Berezhkovskii and G. H. Weiss, Physica A, 2000, 282, 475-485.

34 Y. Jung, E. Barkai and R. J. Silbey, Chem. Phys., 2002, 284, 181-194.

35 R. Verberk and M. Orrit, J. Chem. Phys., 2003, 119, 2214-2222.

36 E. Barkai, Y. J. Jung and R. Silbey, Annu. Rev. Phys. Chem., 2004, 55, 457-507.

37 G. Margolin and E. Barkai, J. Chem. Phys., 2004, 121, 1566-1577.

38 J. Tang and R. A. Marcus, J. Chem. Phys., 2005, 123, 054704.

39 H. Htoon, M. Furis, S. A. Crooker, S. Jeong and V. I. Klimov, Phys. Rev. B: Condens. Matter Mater. Phys., 2008, 77, 035328.

40 V. I. Klimov, J. A. McGuire, R. D. Schaller and V. I. Rupasov, Phys. Rev. B: Condens. Matter Mater. Phys., 2008, 77, 195324.

41 J. J. Peterson and D. J. Nesbitt, Nano Lett., 2009, 9, 338-345.

42 J. Zhao, G. Nair, B. Fisher and M. Bawendi, Phys. Rev. Lett., 2010, 104, 157403.

43 G. Nair, J. Zhao and M. Bawendi, Nano Lett., 2011, 11, 1136-1140.

44 S. L. Sewall, A. Franceschetti, R. R. Cooney, A. Zunger and P. Kambhampati, Phys. Rev. B: Condens. Matter Mater. Phys., 2009, 80, 081310.
45 P. Kambhampati, Acc. Chem. Res., 2011, 44, 1-13.

46 A. A. Cordones, T. J. Bixby and S. R. Leone, J. Phys. Chem. C, 2011, 115, 6341-6349.

47 P. A. Frantsuzov and R. A. Marcus, Phys. Rev. B: Condens. Matter Mater. Phys., 2005, 72, 155321.

48 S. A. Empedocles and M. G. Bawendi, J. Phys. Chem. B, 1999, 103, 1826-1830.

49 G. Margolin, V. Protasenko and M. Kuno, Adv. Chem. Phys., 2006, 133, 327-356.

50 A. Efros and M. Rosen, Phys. Rev. Lett., 1997, 78, 1110-1113.

51 R. Kraus, P. Lagoudakis, J. Müller, A. Rogach, J. Lupton, J. Feldman, D. Talapin and H. Weller, J. Phys. Chem. B, 2005, 109, 18214-18217.

52 B. R. Fisher, H. Eisler, N. E. Stott and M. G. Bawendi, J. Phys. Chem. B, 2003, 108, 143-148.

53 R. A. Marcus, Discuss. Faraday Soc., 1960, 29, 21-31.

54 R. A. Marcus, J. Chem. Phys., 1965, 43, 679-701.

55 G. Schlegel, J. Bohnenberger, I. Potapova and A. Mews, Phys. Rev. Lett., 2002, 88, 137401.

56 For more details on the potential energy surfaces, please refer to ref. 38 and 57.

57 R. A. Marcus, Philos. Trans. R. Soc., A, 2010, 368, 1109-1124.

58 I. Stakgold, Green's functions and boundary value problems, John Wiley, New York, 1979.

59 M. Pelton, G. Smith, N. F. Scherer and R. A. Marcus, Proc. Natl. Acad. Sci. U. S. A., 2007, 104, 14249-14254.

60 C. H. Crouch, O. Sauter, X. Wu, R. Purcell, C. Querner, M. Drndic and M. Pelton, Nano Lett., 2010, 10, 1692-1698.

61 K. T. Early and D. J. Nesbitt, Nano Lett., 2013, 13, 4844-4849.

62 L. P. Watkins and H. Yang, J. Phys. Chem. B, 2005, 109, 617-628.

63 A. P. Beyler, L. F. Marshall, J. Cui, X. Brokmann and M. G. Bawendi, Phys. Rev. Lett., 2013, 111, 177401.

64 C. Javaux, B. Mahler, B. Dubertret, A. Shabaev, A. V. Rodina, A. L. Efros, D. R. Yakovlev, F. Liu, M. Bayer, G. Camps, L. Biadala, S. Buil, X. Quelin and J. P. Hermier, Nat. Nanotechnol., 2013, 8, 206-212.

65 A. Pandey and P. Guyot-Sionnest, J. Phys. Chem. Lett., 2010, 1, 45-47.

66 W. Qin and P. Guyot-Sionnest, ACS Nano, 2012, 6, 9125-9132. 